\title{
Crónica
}

\section{Ministros de Educación y SERNAM lanzan programas de educación sexual y afectividad}

(http://www.mineduc.cl/index2.php?id_contenido $=13695 \&$ id_portal $=1$ \&id_seccion $=10$ )

Ministros de Educación y del Sernam, Joaquín Lavín y Carolina Schmidt, dieron a conocer a la comunidad escolar la forma de optar a los siete programas de educación sexual y afectividad. A partir de hoy 14 de marzo de 2011, los colegios podrán optar a estos programas a través de la página www. mineduc.cl. Se habilitará un fondo especial de $\$ 300$ millones para que los establecimientos más vulnerables puedan acceder a este contenido. También los colegios podrán optar a estos programas a través de los recursos de la subvención escolar preferencial. La implementación de estos programas, incluye además algunos cambios esenciales; "La educación sexual y la afectividad se adelanta y se hace obligatoria para niños más chicos. Hoy día la educación sexual se trata en el curso de ciencias naturales y biología en $7^{\circ}$ básico y $2^{\circ}$ medio. En el futuro será obligatorio en los programas de estudio de primer ciclo básico", explicó el Ministro Lavín. Además, dijo que "se va a crear una hora que se llamará de Orientación, entre $1^{\circ}$ y $4^{\circ}$ básico, en que ya los niños van a ser educados en la afectividad. Ya no se saca nada con enseñar biológicamente este tema cuando los niños están en $7^{\circ}$ básico. Los niños tienen que ser educados de $1^{\circ}$ básico en adelante, en los afectos, en el respeto con su cuerpo y el de los demás, en no dejar que alguien se te acerque o te toque si tú no quieres o en la forma que tú no quieres". Para elegir estos programas de educación sexual, el Ministro de Educación y la Ministra del Sernam formaron una comisión de expertos para crear este portafolio de programas con el objetivo que los establecimientos educacionales puedan elegir el que más les interese o se adecue a su visión y realidad socioeducativa. La comisión fue integrada por el Dr. Sergio Canals, psiquiatra infanto-juvenil, la Dra. Paula Bedregal, PhD en psicología y especialista en medicina familiar del niño y salud pública y Benito Baranda, director del programa Iniciativa América del Hogar de Cristo y Vicepresidente de la Fundación para la Superación de la Pobreza. Estos especialistas utilizaron los siguientes criterios de selección de los programas:

1. Discurso a favor de la postergación del inicio de la actividad sexual de los jóvenes hasta etapas de mayor madurez biopsicosocial.

2. Incorporación de los distintos actores de la Comunidad Educativa, especialmente padres y docentes.

3. Contenidos y metodologías pertinentes con el desarrollo evolutivo de niños, niñas y jóvenes.

4. Contenidos y metodología propuestos en un continuo desde los niveles de básica hasta enseñanza media.

5. Diversidad de enfoques en cuanto a la relación sexualidad-afectividad y control de la natalidad.

6. Visión antropológica clara.

7. Capacidad de difusión y cobertura para implementarse a nivel nacional.

Los programas elegidos son:

1. "Adolescencia: tiempo de decisiones" de la Facultad de Medicina de la Universidad de Chile.

2. "Teen Star" de la Facultad de Ciencias Biológicas de la Universidad Católica de Chile

3. "Sexualidad, Autoestima y Prevención del Embarazo en la Adolescencia" de la Asociación Chilena de Protección de la Familia (APROFA).

4. "Programa de Aprendizaje, Sexualidad y Afectividad (PASA)" de la Facultad de Psicología de la Universidad de Chile.

5. "Programa de Educación en Valores, Afectividad y Sexualidad (PAS) del Centro de Estudios de la Familia de la Universidad San Sebastián.

6. "Aprendiendo a querer" de la Universidad Católica de la Santísima Concepción, Monseñor Ricardo Ezzati.

7. "Curso de Educación Sexual Integral" Dr. Ricardo Capponi (CESI). 